2013-01

Network position: a key component in

the characterization of social personality

types

\title{
Wilson, Alexander
}

http://hdl.handle.net/10026.1/11468

10.1007/s00265-012-1428-y

Behavioral Ecology and Sociobiology

Springer Science and Business Media LLC

All content in PEARL is protected by copyright law. Author manuscripts are made available in accordance with publisher policies. Please cite only the published version using the details provided on the item record or document. In the absence of an open licence (e.g. Creative Commons), permissions for further reuse of content should be sought from the publisher or author. 


\title{
Network position: a key component in the characterization of social personality types
}

\author{
Alexander D. M. Wilson • Stefan Krause • \\ Niels J. Dingemanse $\cdot$ Jens Krause
}

Received: 13 March 2012 /Revised: 3 October 2012 / Accepted: 3 October 2012 /Published online: 16 October 2012

(C) Springer-Verlag Berlin Heidelberg 2012

\begin{abstract}
In recent years, animal social interactions have received much attention in terms of personality research (e.g. aggressive or cooperative interactions). However, other components of social behaviour such as those describing the intensity, frequency, directedness and individual repeatability of interactions in animal groups have largely been neglected. Network analysis offers a valuable opportunity to characterize individual consistency of traits in labile social groups and therein provide novel insights to personality research in ways previously not possible using traditional techniques. Should individual network positions be consistently different between individuals under changing conditions, they might reflect expressions of an individual's personality. Here, we discuss a conceptual framework for
\end{abstract}

Communicated by D. P. Croft

A. D. M. Wilson $(\bowtie) \cdot$ J. Krause

Department of the Biology and Ecology of Fishes,

Leibniz-Institute of Freshwater Ecology and Inland Fisheries,

12587 Berlin, Germany

e-mail: alexander.wilson@ymail.com

J. Krause

Humboldt University,

Berlin, Germany

S. Krause

Department of Electrical Engineering and Computer Science, Lübeck University of Applied Sciences,

23562 Lübeck, Germany

\section{N. J. Dingemanse}

Behavioural Ecology, Department of Biology II,

Ludwig-Maximilians-University of Munich,

Planegg-Martinsried,

Munich, Germany

\section{N. J. Dingemanse}

Evolutionary Ecology of Variation Group,

Max Planck Institute for Ornithology,

Seewiesen, Germany using network analyses to infer the presence of individual differences and present a statistical test based on randomization techniques for testing the consistency of network positions in individuals. The statistical tools presented are useful because if particular individuals consistently occupy key positions in social networks, then this is also likely to have consequences for their fitness as well as for that of others in the population. These consequences may be particularly significant since individual network position has been shown to be important for the transmission of diseases, socially learnt information and genetic material between individuals and populations.

Keywords Social network analysis · Behavioural types · Temperament

Animal personality and social network analysis have been the subject of significant research interest in recent years, and their potential ecological and evolutionary importance has been suggested by numerous empirical and conceptual studies in both fields of research (Réale et al. 2007; Sih et al. 2009; Sih et al. 2012; Wolf and Weissing 2012). Yet in spite of great potential, the integration of these two areas of research remains largely in its infancy (Krause et al. 2010). This situation is due partly to conceptual challenges and partly to difficulties associated with delineating what are generally considered individual traits in dynamic social environments. Animal 'personality' occurs when between-individual variation in behaviour is maintained across contexts (Réale et al. 2007; Dingemanse et al. 2010a). Such between-individual variation has been demonstrated for a wide variety of behaviours (e.g. boldness, activity, aggression and sociability) (Bell et al. 2009). While interest in between-individual variation has existed for some time, new emphases in personality research have been placed on the adaptive mechanisms causing and maintaining such variation (reviewed by Dingemanse and 
Wolf 2010; Wolf and Weissing 2010) and how its presence affects ecological and evolutionary processes (Sih et al. 2012; Wolf and Weissing 2012). Surprisingly, few studies have examined how and why individual repeatability in social behaviours might be maintained, in spite of evidence from the network literature suggesting that network attributes can be heritable (Fowler et al. 2009), related to a variety of ecologically relevant processes (Hamede et al. 2009; Drewe 2010), and hence might evolve in response to selection (Lea et al. 2010). Studies of what are generally considered social traits (i.e. cooperative or agonistic interactions) typically compare individuals dyadically (Wilson et al. 2010b) or on a hierarchical basis (i.e. more or less aggressive), but other aspects of these behavioural phenomena are often overlooked. The relative frequency of interactions, individual preference for particular group members and the potential ecological significance of these characteristics represent just some of the variables often ignored in typical individual-level studies of behavioural traits. Thus, in many respects, personality research on a social or group level has often lacked critical information for understanding the mechanistic underpinnings or adaptive benefit of such consistency in behavioural types (but see Cote et al. 2008; Wilson et al. 2010a).

The recent advent of social network analysis provides a range of statistical tools that allows the characterization of the fine social fine structure of animal groups and populations in a manner previously not possible (Krause et al. 2007; Croft et al. 2008; Wey et al. 2008). Importantly, network analysis provides an investigatory framework for considering not just the intensity of social interactions (i.e. level of expression), but also the consistency and frequency of individual-level interactions and preferential associations in a labile social environment. In this respect, consistency in individual network position may even represent an outcome of individual variation in key behavioural traits (personality). Should this be the case, this notion may have significant ecological and evolutionary implications. For example, individuals may act as 'brokers' of information or keystone figures in the transmission of disease and information in animal groups. While these are terms that are already used in the social network literature on animals in some cases (see Croft et al. 2008 for a review), this is often done without checking some of the crucial underlying assumptions for the characterization of behavioural types (e.g. Dingemanse et al. 2012; Dingemanse and Dochtermann 2012). The most important assumption is likely that individuals repeatedly and consistently occupy the same or similar positions in networks. Yet, most network studies are unreplicated, involving only a single recording session which simply does not allow statements on whether individuals occupy particular positions consistently over time. The aim of this paper is to provide an investigatory framework for considering individual variation in network position and to underscore the potential importance of this level of variation in terms of the ecology and evolution of personality in animals.

\section{Network positions of individuals}

Over the last decade or so, significant advances have been made in the application of network analysis to animal populations. These advances have been, in part, owing to the adaptation and application of network calculations and descriptors from psychology, and also the utilization of new descriptors better suited to the study of animal populations (see Croft et al. 2008). For example, network analysis allows one to examine the social structure of animal groups and provide novel insight into the potential costs and benefits of different positions in a network. Many studies have shown that different positions in animal groups (both spatially and socially) can have distinct advantages and disadvantages in terms of predator avoidance, access to information and disease transmission (reviewed in Krause and Ruxton 2002; Croft et al. 2008). By looking at these advantages with a network perspective, new insight can be gained into important processes underlying social phenomena (i.e. dominance hierarchies) and therein social evolution (Shizuka and McDonald 2012).

For the purposes of this review, we will limit our discussion to several of the most commonly used and ecologically relevant descriptors of network positions (but see Newman 2003; Croft et al. 2008 for additional examples and descriptions). Some of the most promising descriptors (e.g. node betweenness and degree) for application to personality research involve aspects of 'centrality' which generally estimate the social importance of an individual based on the number and frequency of interactions with other group members. An individual's degree is the number of immediate neighbours a particular individual has. Node betweenness, however, measures the extent to which a focal individual lies in shortest paths between pairs of other individuals (Croft et al. 2008). These descriptors are important as they provide a manner by which to characterize and describe ecologically relevant individual-level interactions as well as the importance of particular individuals in population-level social structures. For example, Lusseau and Newman (2004) characterized the social structure of bottlenose dolphins (Tursiops spp.) and suggested that some individuals (as indicated by their high 'betweenness') might act as social brokers by interconnecting otherwise distinct communities. Similarly, other studies have used degree and node betweenness to track disease transmission among captive (e.g. Otterstatter and Thomson 2007) and wild (e.g. Hamede et al. 2009; Drewe 2010) populations as well as to characterize the importance of the number versus nature (who is connected to whom) of connections in animal contact networks. 
Another potentially useful network metric for application to personality research is that of the local clustering coefficient. The local clustering coefficient is a measure of cliquishness derived from local network structure (i.e. phenotypic assortment or associations between familiar individuals). It quantifies the extent to which (immediate) neighbours of some focal individual are themselves neighbours (Croft et al. 2008). A social 'clique' is a subset of highly connected individuals in a network. Cliques and the level of connectedness in a network can be important as the removal of certain key individuals can result in a loss of network cohesion and, perhaps, the collapse of a network that is not well connected. For example, using experimental manipulations of network composition in pig-tailed macaques (Macaca nemestrina), Flack et al. (2006) found that the removal of high-ranking individuals involved in group policing resulted in a notable decline in group cohesion. Additionally, in some cases, cliques may also be a necessary factor in the evolution of cooperative behaviours that may only develop in such highly interconnected groups of individuals in a population.

\section{Between-individual variation in network position}

The use of network positions provides a unique opportunity to describe important new social aspects of personality that has thus far been omitted from studies of behavioural types. However, despite the obvious potential for the application of network analysis to personality research (Krause et al. 2010), surprisingly few studies have attempted to characterize such a relationship (Pike et al. 2008; Croft et al. 2009), and fewer still have described network position in terms of personality (but see Lusseau and Newman 2004). The importance of this notion lies in the fact that network positions may reflect important ecological and evolutionary processes (Otterstatter and Thomson 2007; Fowler et al. 2009; Hamede et al. 2009; Krause et al. 2010). Some network positions may have higher fitness than others and represent significant differences in the investment of time and resources (see Krause et al. 2010). For example, if one were to consider connectedness as above, a highly connected individual may be required to devote considerably more time and energy in establishing and maintaining connections than a poorly connected individual. This differential allocation of resources may have significant costs (or benefits as the case may be) in terms of mating, competition, foraging and antipredator behaviour, but will likely vary between networks and the nature of the social ties between individuals. While a given network position might be stable over the long term, acute changes in ambient conditions (i.e. competition and disease) might result in short-term changes in network position optima when involving seasonal considerations (i.e. breeding cycles) or perhaps more longlasting changes resulting from ontogenetic processes or niche shifts (sensu Wilson and Krause 2012a, b). In humans, maintaining high levels of connections among individuals using social media networks (e.g. Facebook) has been shown to result in the formation and maintenance of 'social capital', which may be considered a surrogate term for the acquisition of new information and resources between individuals (Ellison et al. 2007). Further, as with other consistent individual differences in behaviour, network positions as traits may coevolve with other important behavioural and/ or morphological traits (Sih et al. 2004). Our proposed novel approach in using network position in studies of personality would therein provide and integrate a wealth of quantitative variables (type, frequency and intensity) regarding social interactions where previous studies were limited to dyadic relationships.

There are some conceptual challenges associated with the use of network position in personality research, not least of which is delineating the behaviour exhibited by a focal individual when it is not independent of the behaviour exhibited by others (Webster and Ward 2011). However, so long as the relative differences between individuals remain consistent, the influence of social context on the absolute value of an individual's average trait expression is not overtly important for considering repeatable differences in network position. The use of appropriate network descriptors and testing for the consistency of network positions between networks (see statistical test below) may open up many new experimental possibilities for research into animal personality traits and their proximate and evolutionary bases.

\section{Network metrics}

In this study, we have utilized three of the most commonly used node-based network metrics as outlined earlier in this paper, that of degree, node betweenness and local clustering coefficient. However, we have provided more detailed definitions and calculations of our descriptors and related network factors for the sake of clarity.

A network consists of a set of 'nodes' or 'vertices' $V=$ $\left\{v_{1}, v_{2}, \ldots, v_{n}\right\}$ and a set of 'edges' $E=\left\{e_{1}, e_{2}, \ldots, e_{m}\right\}$, each of which connects two nodes. There are different types of networks, for example directed and undirected networks. In a directed network, each edge has a direction and a directed edge $e=\left(v_{x}, v_{y}\right)$ connects $v_{x}$ to $v_{y}$, but not $v_{y}$ to $v_{x}$. In contrast, in an undirected network, each edge connects its nodes in both directions. Also, networks may be weighted or unweighted. In a weighted network, each edge is assigned a number which is called its 'weight'. Which type of network is the most appropriate one depends on the type of relation that is modelled by the network. For relations that are not 
symmetric, it is advantageous to use directed networks. If it is important to express that the connections have different 'strength' or 'length', weighted networks should be used.

In this study, we use undirected, unweighted networks without self-loops (i.e. edges that connect a node to itself). Such networks are usually called 'simple' networks. Note that most of the definitions in this section only apply to this type of network. However, the definitions can be modified and extended such that weighted, directed edges can be taken into account (see Croft et al. 2008, for examples). For a focal node $v_{1}$, the nodes that are connected to $v_{1}$ via some edge are called the 'neighbours' of $v_{1}$. For our purposes, we will describe the descriptors we are measuring in our statistical test as (1) degree, (2) node betweenness and (3) local clustering coefficient.

Firstly, the degree of a node is simply the number of its neighbours. The possible range of values of the degree depends on the number of nodes in the network. In an undirected network with $N$ nodes, the degree of each node is in the range $0 \ldots N-1$.

Secondly, the node betweenness of a node $v$ measures the extent to which shortest paths between other nodes run through $v$. A path between two nodes $v_{\mathrm{s}}$ and $v_{\mathrm{e}}$ is a sequence of edges that connect $v_{\mathrm{s}}$ to $v_{\mathrm{e}}$ via zero or more intermediate nodes. A shortest path between two nodes $v_{\mathrm{s}}$ and $v_{\mathrm{e}}$ in an unweighted network is a path with a minimal number of edges. Note that there may be more than one shortest path between two nodes. If $b\left(v_{s}, v_{f}, v_{e}\right)$ is the proportion of shortest paths between $v_{\mathrm{s}}$ and $v_{\mathrm{e}}$ that run through a focal node $v_{\mathrm{f}}$, then the node betweenness of $v_{f}$ in an undirected network is the sum of the proportions $b\left(v_{i}, v_{f}, v_{j}\right)$ of $v_{f}$ regarding all pairs of other nodes $v_{i}$ and $v_{j}$. More formally,

Node betweenness $\left(v_{f}\right)=\Sigma_{i<j, i \neq f, j \neq f} b\left(v_{i}, v_{f}, v_{j}\right)$.

Lastly, the local clustering coefficient of a node $v$ is the proportion of possible edges between neighbours of $v$ that actually occur in the network. More formally, if the node $v$ has $k$ neighbours, and there exist $m$ edges between these neighbours, then

Local clustering coefficient $(v)=2 m /(k(k-1))$, if

$k>1$, and 0 otherwise.

By definition, the local clustering coefficient is always in the range $0 \ldots 1$.

\section{Testing individual consistency in network position}

To test whether individuals show repeatable differences in network position, it is critical to have some measure of consistency and repeatability of network position over time and/or situations. This requires repeated observations of a given group or population of individuals and tracking of all individuals within that network over a set time period or number of observations. If a population is sampled over multiple sessions, and for each session the social network structure is established, a randomization test can be applied to check for consistency of network positions of the individuals. We will explain the details of such a test in this section. To illustrate our test, we use networks that were generated by a very simple simulation programme, which will be explained in the next section.

In a network, the node values for a given network measure are not independent of each other. Therefore, we use a randomization test. We assume that we have $K$ networks each of which was established by sampling the same population of $N$ individuals multiple times. We construct a null model by permuting the node labels in each network, and we use as a test statistic the sum of the variances of individual ranks in the networks (SV). In the case of tied values, we use mean ranks. More precisely, if $R$ is a matrix of ranks, where $R_{i, k}$ is the rank of individual $i$ in network $k$ regarding a certain network measure, then the test statistic $\mathrm{SV}$ is defined as follows: $\operatorname{SV}(R)=\sum_{1 \leq i \leq N} \operatorname{variance}\left(R_{i, 1}, \ldots R_{i, K}\right)$, where $N$ is the number of individuals (= number of rows) and $K$ the number of networks (= number of columns).

The main reason for using ranks rather than raw values is that we are interested in individual network positions relative to other individuals. In this context, it is more important to find out, for example, whether certain individuals tend to have a relatively high node betweenness compared to others than whether individuals tend to have similar concrete node betweenness values. Also, while it is obvious that for each individual all ranks are equally probable under the null model, we usually do not know very much about the distribution of the raw values unless we have full knowledge of the mechanism that generates the networks. A test statistic that measures the variability of the raw values would therefore be difficult or even impossible to interpret without this knowledge, despite parametric metrics having obvious heuristic values in evolutionary research (Dingemanse et al. 2012a).

If individuals have similar ranks across the observed networks, the test statistic should yield small values. Following the usual definition of randomization tests (Manly 2007) for a given level $\alpha$, we regard the value $\mathrm{SV}\left(R_{1}\right)$ of our observed matrix $R_{1}$ as significant, if it is among the $m \times \alpha$ smallest values of the sequence $\mathrm{SV}\left(R_{1}\right), \mathrm{SV}\left(R_{2}\right), \ldots \mathrm{SV}\left(R_{m}\right)$ of $m$ values, where $\mathrm{SV}\left(R_{2}\right), \ldots, \mathrm{SV}\left(R_{m}\right)$ are constructed by permuting the values of each column of $R_{1}$.

Note that there are two different sources of consistency of network positions. Firstly, individuals may have certain preferences when choosing a group member to interact with. For example, individuals that strongly prefer to interact with only a small number of their group members are likely to 
have a consistently lower degree than individuals who have no specific preferences. Secondly, individuals may have different tendencies to interact at all. For example, even if no individual in a population has specific preferences for other members, individuals with higher interaction frequencies will have higher degrees than others. Therefore, it is important that no bias is introduced by the observation method. More precisely, the probability of observing an individual must be equal for all individuals. Otherwise, there is a risk of reporting spurious interpretations. If for some individuals it is known that they have been observed with lower probability, the bias can be alleviated by filtering the networks, i.e. by removing these individuals and all their edges from the networks, though such an approach would possibly also result in a biased estimate of the population if the removed individuals are of specific behavioural type.

\section{Illustrative example}

To illustrate our randomization test, we simulated observation sessions of a population of $N$ interacting individuals in a very simple way that allows one to distinguish between the two sources of consistency mentioned above. We used the programming language Java and the Java Universal Network/Graph Framework JUNG version 2.0.1 (Madadhain et al. 2005).

In our simulation, each individual will be involved in a fixed number of interactions and each individual has specific preferences for the other individuals. For individual $i$, the number of interactions is denoted by $a_{i}$ and the preference for individual $j$ by $p_{i}(j)$. More precisely, the simulation of an observation session works as follows. $M=\sum_{1<i<N} a_{i} / 2$ times an individual $i$ is picked, and for this individual, a partner $j$ is chosen with probability $p_{i}(j)$, where $\sum_{1 \leq j \leq N}$ $p_{i}(j)=1$ and $p_{i}(i)=0$, such that the number of interactions of neither $i$ nor $j$ exceeds the limits $a_{i}$ and $a_{j}$, respectively. After an observation $S$ consisting of $M$ interactions has been generated, a network is constructed by adding an edge $(i, j)$ for each interaction between the individuals $i$ and $j$ contained in $S$. This means that the resulting network will contain at most $M$ edges and each individual will have a degree of at most $a_{i}$.

If all $a_{i}$ and all $p_{i}(j)$ are equal, respectively, for each individual, all ranks (regarding any network metric) are equally probable, and for a set of $K$ networks constructed from separate simulated observation sessions of the same population, the consistency test will reject the null hypothesis in $100 \%$ of the cases. If, however, the $a_{i}$ or the $p_{i}(j)$ differ, some individuals will tend to occupy certain network positions with a higher probability than others. In our example, we chose the same number of interactions $\left(a_{i}=6\right)$ for all individuals $(N=$ $15)$, but different individual preferences $p_{i}(j)$ to generate a set of five networks (Fig. 1). The values of $p_{i}(j)$ are shown in Table 1. Here, some individuals tend to form clusters, while others choose their partners more randomly.

Using the randomization test described above, we can demonstrate how different measures of individual network positions can be consistent over five observation sessions when using degree $(p=0.031, \mathrm{SV}=184.2$, Table 2$)$, the local clustering coefficient $(p=0.009, \mathrm{SV}=204.8$, Table 3$)$ and node betweenness $(p=0.003, \mathrm{SV}=202.0$, Table 4$)$ as network descriptors (following $10^{4}-1$ randomizations). Of course, our test will not detect consistency of network positions in every set of networks generated using our example probabilities. The percentage of detections strongly depends on the number of interactions $a_{i}$. For example, given the preferences in Table 1, in 10,000 simulated sets of five networks, the test (with $10^{4}-1$ randomizations, $\alpha=$ 0.05 ) found consistency regarding the node betweenness in $10.3 \%$ of the cases for $a_{i}=2$ (i.e. if only two interactions per individual were simulated), in $39.6 \%$ of the cases for $a_{i}=6$ and in $90.1 \%$ of the cases for $a_{i}=12$. (a)

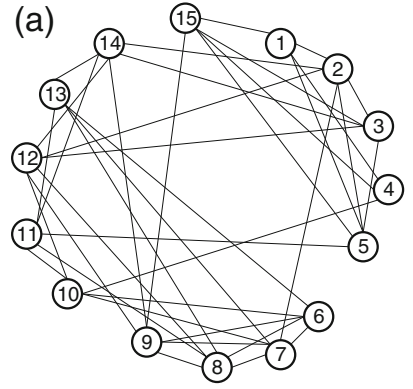

(c)
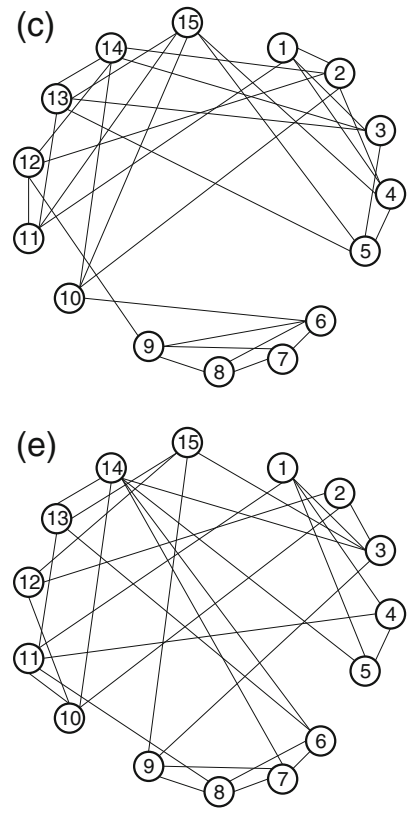

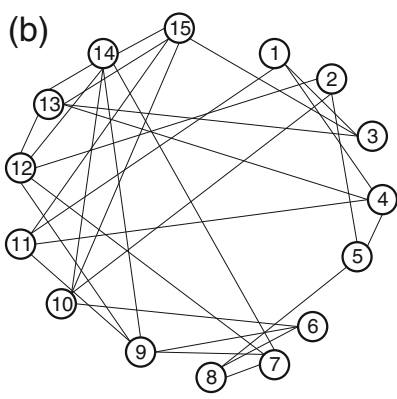

(d)

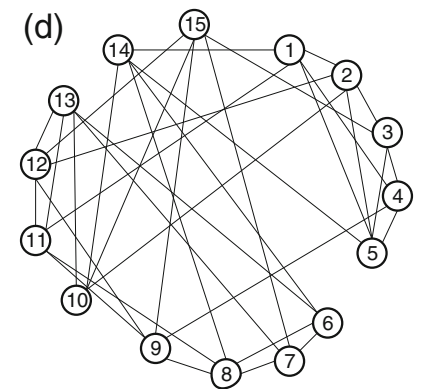

Fig. 1 Social networks (a-e) generated from the same fictitious population of 15 individuals. Numbered nodes represent individuals and edges (lines) social connections between them 
Table 1 Probabilities $p_{i}(j)$ used in the network simulation to express individual preferences (for example, the value 0.22 in line 3 , column 4 means that when individual 3 interacts, it will choose individual 4 with probability 0.22 )

\begin{tabular}{|c|c|c|c|c|c|c|c|c|c|c|c|c|c|c|c|c|}
\hline & & \multicolumn{15}{|c|}{ Individual $j$} \\
\hline & & 1 & 2 & 3 & 4 & 5 & 6 & 7 & 8 & 9 & 10 & 11 & 12 & 13 & 14 & 15 \\
\hline \multirow[t]{15}{*}{ Individual $i$} & 1 & 0 & 0.22 & 0.22 & 0.22 & 0.22 & 0.01 & 0.01 & 0.01 & 0.01 & 0.01 & 0.01 & 0.01 & 0.01 & 0.02 & 0.02 \\
\hline & 2 & 0.22 & 0 & 0.22 & 0.22 & 0.22 & 0.01 & 0.01 & 0.01 & 0.01 & 0.01 & 0.01 & 0.01 & 0.01 & 0.02 & 0.02 \\
\hline & 3 & 0.22 & 0.22 & 0 & 0.22 & 0.22 & 0.01 & 0.01 & 0.01 & 0.01 & 0.01 & 0.01 & 0.01 & 0.01 & 0.02 & 0.02 \\
\hline & 4 & 0.22 & 0.22 & 0.22 & 0 & 0.22 & 0.01 & 0.01 & 0.01 & 0.01 & 0.01 & 0.01 & 0.01 & 0.01 & 0.02 & 0.02 \\
\hline & 5 & 0.22 & 0.22 & 0.22 & 0.22 & 0 & 0.01 & 0.01 & 0.01 & 0.01 & 0.01 & 0.01 & 0.01 & 0.01 & 0.02 & 0.02 \\
\hline & 6 & 0.01 & 0.01 & 0.01 & 0.01 & 0.01 & 0 & 0.29 & 0.29 & 0.29 & 0.01 & 0.01 & 0.01 & 0.01 & 0.02 & 0.02 \\
\hline & 7 & 0.01 & 0.01 & 0.01 & 0.01 & 0.01 & 0.29 & 0 & 0.29 & 0.29 & 0.01 & 0.01 & 0.01 & 0.01 & 0.02 & 0.02 \\
\hline & 8 & 0.01 & 0.01 & 0.01 & 0.01 & 0.01 & 0.29 & 0.29 & 0 & 0.29 & 0.01 & 0.01 & 0.01 & 0.01 & 0.02 & 0.02 \\
\hline & 9 & 0.01 & 0.01 & 0.01 & 0.01 & 0.01 & 0.29 & 0.29 & 0.29 & 0 & 0.01 & 0.01 & 0.01 & 0.01 & 0.02 & 0.02 \\
\hline & 10 & 0.01 & 0.40 & 0.01 & 0.01 & 0.01 & 0.40 & 0.01 & 0.01 & 0.01 & 0 & 0.01 & 0.01 & 0.01 & 0.04 & 0.06 \\
\hline & 11 & 0.30 & 0.02 & 0.02 & 0.02 & 0.02 & 0.02 & 0.02 & 0.30 & 0.02 & 0.02 & 0 & 0.06 & 0.06 & 0.06 & 0.06 \\
\hline & 12 & 0.02 & 0.30 & 0.02 & 0.02 & 0.02 & 0.02 & 0.02 & 0.02 & 0.30 & 0.02 & 0.06 & 0 & 0.06 & 0.06 & 0.06 \\
\hline & 13 & 0.07 & 0.07 & 0.07 & 0.07 & 0.07 & 0.07 & 0.07 & 0.07 & 0.07 & 0.07 & 0.07 & 0.07 & 0 & 0.07 & 0.09 \\
\hline & 14 & 0.07 & 0.07 & 0.07 & 0.07 & 0.07 & 0.07 & 0.07 & 0.07 & 0.07 & 0.07 & 0.07 & 0.07 & 0.07 & 0 & 0.09 \\
\hline & 15 & 0.07 & 0.07 & 0.07 & 0.07 & 0.07 & 0.07 & 0.07 & 0.07 & 0.07 & 0.07 & 0.07 & 0.07 & 0.07 & 0.09 & 0 \\
\hline
\end{tabular}

\section{Additional analyses}

In our example, the individual mean ranks were correlated across the network descriptors (all two-sided $p$ values $<0.035$ in randomization tests with 9,999 randomizations and Kendall's $\tau$ as a test statistic). In real networks, this is not necessarily the case. However, it is to be expected that some descriptors might occasionally be correlated, and as such, it is important to determine which traits are independent and which covary. Correlation analysis provides a potential starting point for characterizing network position traits by relating independent descriptors to each other and other traits and therein assists in the delineation of the properties of a network; its structure could be further explored using multivariate methods detailed

Table 2 Individual differences in degree for a fictitious population $(N=15)$ over five simulated sampling sessions

\begin{tabular}{|c|c|c|c|c|c|c|c|c|c|c|c|c|}
\hline \multirow[t]{2}{*}{ Individual } & \multicolumn{2}{|c|}{ Sample 1} & \multicolumn{2}{|c|}{ Sample 2} & \multicolumn{2}{|c|}{ Sample 3} & \multicolumn{2}{|c|}{ Sample 4} & \multicolumn{2}{|c|}{ Sample 5} & \multirow[t]{2}{*}{ Variance of ranks } & \multirow[t]{2}{*}{ Mean rank } \\
\hline & Value & Rank & Value & Rank & Value & Rank & Value & Rank & Value & Rank & & \\
\hline 1 & 4 & 14 & 3 & 12.5 & 4 & 9 & 5 & 5.5 & 4 & 7.5 & 12.3 & 9.7 \\
\hline 2 & 6 & 3.5 & 3 & 12.5 & 5 & 2.5 & 5 & 5.5 & 3 & 13.5 & 26.5 & 7.5 \\
\hline 3 & 5 & 10 & 3 & 12.5 & 4 & 9 & 4 & 13 & 5 & 2.5 & 17.7 & 9.4 \\
\hline 4 & 3 & 15 & 4 & 7.5 & 4 & 9 & 4 & 13 & 3 & 13.5 & 10.2 & 11.6 \\
\hline 5 & 5 & 10 & 3 & 12.5 & 4 & 9 & 5 & 5.5 & 3 & 13.5 & 9.9 & 10.1 \\
\hline 6 & 5 & 10 & 3 & 12.5 & 4 & 9 & 4 & 13 & 4 & 7.5 & 5.4 & 10.4 \\
\hline 7 & 6 & 3.5 & 4 & 7.5 & 3 & 14.5 & 4 & 13 & 4 & 7.5 & 20.2 & 9.2 \\
\hline 8 & 6 & 3.5 & 3 & 12.5 & 3 & 14.5 & 5 & 5.5 & 4 & 7.5 & 21.7 & 8.7 \\
\hline 9 & 6 & 3.5 & 5 & 3.5 & 4 & 9 & 5 & 5.5 & 4 & 7.5 & 6.0 & 5.8 \\
\hline 10 & 5 & 10 & 4 & 7.5 & 4 & 9 & 4 & 13 & 4 & 7.5 & 5.2 & 9.4 \\
\hline 11 & 5 & 10 & 4 & 7.5 & 4 & 9 & 5 & 5.5 & 5 & 2.5 & 8.9 & 6.9 \\
\hline 12 & 6 & 3.5 & 5 & 3.5 & 4 & 9 & 5 & 5.5 & 3 & 13.5 & 18.3 & 7.0 \\
\hline 13 & 5 & 10 & 5 & 3.5 & 5 & 2.5 & 5 & 5.5 & 4 & 7.5 & 9.2 & 5.8 \\
\hline 14 & 6 & 3.5 & 6 & 1 & 5 & 2.5 & 5 & 5.5 & 6 & 1 & 3.6 & 2.7 \\
\hline 15 & 5 & 10 & 5 & 3.5 & 5 & 2.5 & 5 & 5.5 & 4 & 7.5 & 9.2 & 5.8 \\
\hline
\end{tabular}

Each individual was assigned an actual value and then ranked according to that value for each network observation 
Table 3 Individual differences in the local clustering coefficient for a fictitious population $(N=15)$ over five simulated sampling sessions

\begin{tabular}{|c|c|c|c|c|c|c|c|c|c|c|c|c|}
\hline \multirow[t]{2}{*}{ Individual } & \multicolumn{2}{|c|}{ Sample 1} & \multicolumn{2}{|c|}{ Sample 2} & \multicolumn{2}{|c|}{ Sample 3} & \multicolumn{2}{|c|}{ Sample 4} & \multicolumn{2}{|c|}{ Sample 5} & \multirow[t]{2}{*}{ Variance of ranks } & \multirow[t]{2}{*}{ Mean rank } \\
\hline & Value & Rank & Value & Rank & Value & Rank & Value & Rank & Value & Rank & & \\
\hline 1 & 0.500 & 3 & 0.333 & 4.5 & 0.167 & 13.5 & 0.300 & 8 & 0.333 & 6.5 & 16.4 & 7.1 \\
\hline 2 & 0.333 & 10 & 0.000 & 13.5 & 0.300 & 10 & 0.200 & 12 & 0.333 & 6.5 & 6.9 & 10.4 \\
\hline 3 & 0.500 & 3 & 0.333 & 4.5 & 0.333 & 7.5 & 0.333 & 4 & 0.100 & 14.5 & 21.8 & 6.7 \\
\hline 4 & 0.333 & 10 & 0.167 & 10 & 0.333 & 7.5 & 0.333 & 4 & 0.667 & 1 & 15.5 & 6.5 \\
\hline 5 & 0.400 & 6.5 & 0.000 & 13.5 & 0.500 & 4 & 0.500 & 1.5 & 0.333 & 6.5 & 20.1 & 6.4 \\
\hline 6 & 0.600 & 1 & 0.000 & 13.5 & 0.500 & 4 & 0.500 & 1.5 & 0.500 & 2.5 & 26.6 & 4.5 \\
\hline 7 & 0.400 & 6.5 & 0.500 & 1 & 1.000 & 1.5 & 0.333 & 4 & 0.500 & 2.5 & 4.9 & 3.1 \\
\hline 8 & 0.467 & 5 & 0.000 & 13.5 & 1.000 & 1.5 & 0.300 & 8 & 0.333 & 6.5 & 19.4 & 6.9 \\
\hline 9 & 0.333 & 10 & 0.300 & 7 & 0.500 & 4 & 0.300 & 8 & 0.333 & 6.5 & 4.8 & 7.1 \\
\hline 10 & 0.100 & 15 & 0.167 & 10 & 0.167 & 13.5 & 0.000 & 15 & 0.167 & 11 & 5.3 & 12.9 \\
\hline 11 & 0.200 & 14 & 0.167 & 10 & 0.167 & 13.5 & 0.300 & 8 & 0.100 & 14.5 & 8.1 & 12.0 \\
\hline 12 & 0.333 & 10 & 0.400 & 2.5 & 0.167 & 13.5 & 0.300 & 8 & 0.333 & 6.5 & 16.7 & 8.1 \\
\hline 13 & 0.500 & 3 & 0.300 & 7 & 0.400 & 6 & 0.200 & 12 & 0.167 & 11 & 13.7 & 7.8 \\
\hline 14 & 0.333 & 10 & 0.400 & 2.5 & 0.300 & 10 & 0.200 & 12 & 0.133 & 13 & 17.0 & 9.5 \\
\hline 15 & 0.300 & 13 & 0.300 & 7 & 0.300 & 10 & 0.100 & 14 & 0.167 & 11 & 7.5 & 11.0 \\
\hline
\end{tabular}

Each individual was assigned an actual value and then ranked according to that value for each network observation

elsewhere, particularly factor analysis (Budaev 2010) in combination with structural equation modelling (Dochtermann and Jenkins 2007; Dingemanse et al. 2010a; Dochtermann and Jenkins 2011). Some authors have used mixed models to analyse network data (Otterstatter and Thomson 2007; Frere et al. 2010; Lea et al. 2010). While it is possible to account for some aspects of non-independence of individuals in networks using such models, it remains an open question whether this is generally the case (Croft et al. 2011). The use of mixed models (instead of randomizations) may depend on the response variables (i.e. network metrics) in question and the biological inferences that authors wish to make from the test results.

Table 4 Individual differences in node betweenness for a fictitious population $(N=15)$ over five simulated sampling sessions

\begin{tabular}{|c|c|c|c|c|c|c|c|c|c|c|c|c|}
\hline \multirow[t]{2}{*}{ Individual } & \multicolumn{2}{|c|}{ Sample 1} & \multicolumn{2}{|c|}{ Sample 2} & \multicolumn{2}{|c|}{ Sample 3} & \multicolumn{2}{|c|}{ Sample 4} & \multicolumn{2}{|c|}{ Sample 5} & \multirow[t]{2}{*}{ Variance of ranks } & \multirow[t]{2}{*}{ Mean rank } \\
\hline & Value & Rank & Value & Rank & Value & Rank & Value & Rank & Value & Rank & & \\
\hline 1 & 2.03 & 14 & 1.87 & 15 & 2.50 & 11 & 6.00 & 8 & 5.75 & 6 & 14.7 & 10.8 \\
\hline 2 & 8.79 & 1 & 3.83 & 12 & 11.03 & 6 & 6.35 & 4 & 1.53 & 14 & 29.8 & 7.4 \\
\hline 3 & 2.53 & 11 & 2.23 & 14 & 2.37 & 12 & 3.65 & 12 & 13.88 & 3 & 18.3 & 10.4 \\
\hline 4 & 2.33 & 12 & 10.03 & 3 & 2.87 & 10 & 3.09 & 14 & 0.75 & 15 & 22.7 & 10.8 \\
\hline 5 & 4.42 & 9 & 7.33 & 9 & 1.50 & 13 & 4.60 & 11 & 2.83 & 10 & 2.8 & 10.4 \\
\hline 6 & 1.87 & 15 & 3.25 & 13 & 13.67 & 3 & 2.23 & 15 & 1.75 & 13 & 25.2 & 11.8 \\
\hline 7 & 5.96 & 7 & 5.00 & 10 & 0.00 & 14.5 & 3.12 & 13 & 2.28 & 11 & 8.3 & 11.1 \\
\hline 8 & 3.34 & 10 & 4.00 & 11 & 0.00 & 14.5 & 6.22 & 5 & 5.03 & 9 & 11.8 & 9.9 \\
\hline 9 & 7.95 & 3 & 10.28 & 1 & 11.50 & 5 & 7.60 & 3 & 5.58 & 7 & 5.2 & 3.8 \\
\hline 10 & 8.46 & 2 & 7.58 & 7 & 20.17 & 1 & 5.32 & 10 & 10.40 & 4 & 13.7 & 4.8 \\
\hline 11 & 6.75 & 4 & 8.72 & 6 & 6.40 & 8 & 5.98 & 9 & 14.65 & 2 & 8.2 & 5.8 \\
\hline 12 & 6.08 & 6 & 7.37 & 8 & 17.83 & 2 & 6.04 & 7 & 1.90 & 12 & 13.0 & 7.0 \\
\hline 13 & 2.09 & 13 & 10.22 & 2 & 3.73 & 9 & 6.11 & 6 & 5.42 & 8 & 16.3 & 7.6 \\
\hline 14 & 5.70 & 8 & 8.73 & 5 & 11.73 & 4 & 10.48 & 1 & 18.80 & 1 & 8.7 & 3.8 \\
\hline 15 & 6.69 & 5 & 9.55 & 4 & 10.70 & 7 & 9.22 & 2 & 6.43 & 5 & 3.3 & 4.6 \\
\hline
\end{tabular}

Each individual was assigned an actual value and then ranked according to that value for each network observation 


\section{Logistical and experimental constraints}

Beyond characterizing individual-level consistency in network attributes, a number of logistical and conceptual challenges remain for quantifying individual variation in network position. Logistical challenges include understanding how test power changes with observed group/population size, how often groups/populations should be sampled and how many observations should be made to construct a network per sampling session. Sample size limitations represent a significant issue for work on social networks. Networks that are large (consisting of hundreds or thousands of individuals) are difficult to quantify, manipulate and replicate. Similarly, identifying and tracking individuals, particularly in the wild or with large species or populations, represent further technical challenges. Avoiding recording errors (i.e. mis-identifying individuals) and successfully providing continuous tracking of large numbers of individuals over short observation sessions are also challenging concerns; however, these issues may be partially overcome using technological innovations (Krause et al. 2011). That said, our example demonstrates that consistency in network descriptors may be achieved in as few as 15 individuals, and thus, the problems arising in large populations may be avoided by utilizing smaller groups or populations when possible.

Another issue that needs to be resolved is that each individual's estimate of its 'average' network position comes with uncertainty that should be accounted for when this value is used in further analyses (see Hadfield et al. 2010 for discussion). Modern statistical methods, fortunately, do allow for such uncertainty to be taken forward into followup analyses, for example by using Markov chain Monte Carlo methods and the posterior distributions of estimates (see Dingemanse et al. 2012a).

Experimental challenges include, but are not limited to, finding suitable ways to test for the underlying proximate bases of any consistency in network position, how network position might carry over between different behavioural contexts and how best to deal with populations that frequently change in their social composition (e.g. fissionfusion). Testing individual consistency in network position across networks (via manipulation and/or exchange experiments) may provide an important insight into the plasticity of network position between individuals as well as their adaptive significance in terms of ecology and evolution. Recent studies have highlighted the important role that plasticity has in terms of guiding an individual's overall behavioural phenotype when it comes to personality (Dingemanse et al. 2010b). Since individuals vary in the average level of behaviour they display across different contexts, traits are arguably most interesting when they carryover irrespective of environmental variation. Thus, manipulation/exchange experiments demonstrating that particular individuals retain their relative network position when transferred between different social groups or environments would be a valuable finding for characterizing whether individual network characteristics are maintained (i.e. repeatable) over contexts. Potential applications of this type of exchange experiment may involve transferring individuals possessing similar network properties between groups or exchanging individuals from opposite network positions between groups (e.g. individual of high degree and betweenness exchanged for an individual of low degree and betweenness). The combination of these two types of manipulation experiment would provide interesting insights into the importance of individual behavioural predisposition relative to the ambient social environment in network personality experiments as well as test the robustness of such parameters to changes in group composition and behavioural context. While consistency in network position would be interesting in itself with regard to personality, consistency following disturbance would be especially compelling as individuals might be expected to alter their behaviour in an adaptive manner when confronted with dramatically different social environments.

In contrast, understanding what types of changes in social environment might instead disrupt any potential observed consistency in network position is also invaluable. Several experimental studies have documented evidence of such changes in social dynamics when group composition was changed by the addition of new individuals (Darden et al. 2009; Jacoby et al. 2010) or manipulations based on behavioural type (Sih and Watters 2005). These further investigations into the social dynamics of personality in networks provide valuable novel insight into understanding how the importance of individual network attributes may be affected over different time scales and their robustness to change in dynamic environments. Such studies may also provide important information for studying those species in which animal groups undergo fission-fusion changes in their membership. For example, while our test is ideal for studying network position in captive or closed populations, it is also useful for considering fission-fusion populations as one can modify their sampling technique over longer periods of time to monitor which individuals leave or rejoin and how often groups of individuals are observed together. If certain members of a group are generally observed for similar observation frequencies, standard filtering techniques can exclude transient members and allow analysis of more long-term members. While this is somewhat speculative, these populations would likely need to be considered on a case-by-case basis. That said, the aim of this case study is to provide a novel and useful analytical framework for considering network position in animal groups, and while fissionfusion populations remain a challenge, our test is of clear value for laboratory studies or those studies of wild populations where group membership is relatively constant. 


\section{Fitness consequences of a network position trait}

A key challenge of assessing the fitness consequences of different network positions is to experimentally ascertain how the potential factors affecting network position influence their heritability. While this can be difficult to demonstrate empirically, one approach might involve establishing a causal link between consistent differences in behaviour (i.e. personality) and individual position within a given social group. For example, one might explore the relationship between aggression (a personality trait) and dominance hierarchies (an outcome of interactions between individuals in a group) (McGhee and Travis 2010) and relate this to differences in correlated heritable traits (Moore 1990; Horne and Ylonen 1998).

If network attributes can be repeatable or heritable (Fowler et al. 2009) and individuals consistently occupy particular network positions, then the question arises what the fitness consequences of such positions are and whether selection can shape the evolution of networks. Two plausible scenarios seem likely. First, individuals in different network positions may achieve different absolute fitness values. Should this be the case, how then would these differences arise and be maintained in animal groups and what ecological factors enable certain individuals to have high fitness and others not? Alternatively, individuals in different network positions may initially be capable of achieving the same fitness, but differences in network position may, for whatever reason, be related to differences in other indirect fitness components (e.g. mortality risk and fecundity profile). Not many studies have commented on the fitness consequences of network position, but there are notable exceptions. For example, Oh and Badyaev (2010) found that male house finches (Carpodacus mexicanus) can choose their social background to enhance their attractiveness to female conspecifics. Males with less elaborate ornamentation tended to be more socially labile (showing higher betweenness) relative to more elaborate males (with more colourful plumage). Social labiality was reflected in duller males changing associations between distinct social groups to find groups which best suited their duller ornamentation and therein provide themselves with the maximum possible fitness advantage by increasing their relative attractiveness to females. In another study, McDonald (2007) demonstrated that centrality was a good predictor of future reproductive success and adult social status for juvenile male long-tailed manakins (Chiroxiphia linearis). Ryder et al. (2008) found a similar result in that social network connectivity (as measured by degree, eigenvector centrality, information centrality and reach) predicted the ability of male wire-tailed manakins (Pipra filicauda) to become territory holders and therein obtain greater reproductive success via preferential access to potential mates. However, all of these studies present only correlational evidence and research is now needed to determine whether there is a causal relationship between network position and fitness. Nevertheless, this evidence presents an interesting starting point for an investigation of a link between social structure and function.

\section{Conclusions}

The use of network position in personality research holds much promise for behavioural biologists (Krause et al. 2010). While previously qualitative aspects of animal social interactions have received much attention in terms of personality research (e.g. aggressive or cooperative interactions), until the advent of network analysis, no proper analytic framework existed to describe the quantitative aspects of animal social behaviour (i.e. the intensity, frequency, directedness and consistency of interactions) in a meaningful way. Thus, key components of our understanding of behavioural types and their relationship to group-level dynamics have largely been neglected. The analysis of individual variation in network position meet these demands and also provide a framework for measuring individual-level consistency in animal groups. Since network position has been shown to be important in terms of the transmission of diseases, socially learnt information and genetic material between individuals and populations, it is probable that different network positions may have diverse fitness consequences (Franz and Nunn 2009; Krause et al. 2010). Some network positions may require considerable input of time and resources to attain and/or maintain and thus be associated with significantly different costs and benefits due to this differential allocation of resources between individuals. Understanding how and why these differences arise may provide important insight into the evolution of social personality traits and behavioural types in general. This is particularly important in that recent evidence suggests that at least some network attributes may be heritable and thus subject to selection (Fowler et al. 2009). The statistical test which we developed demonstrates that individual-level consistency in network position can be successfully characterized at the level of the group and under changing social conditions. As such, this technique should represent a valuable tool for those interested in the origin and maintenance of social personality traits and an important next step in the integration of network analysis and personality research.

Acknowledgments We would like to thank Max Wolf and Dick James for helpful discussions and suggestions for the manuscript. ADMW was supported by a postdoctoral research fellowship from the Alexander von Humboldt foundation. NJD was supported by the Max Planck Society. We would also like to thank Darren Croft and two anonymous reviewers for comments and suggestions. 


\section{References}

Bell AM, Hankison SJ, Laskowski KL (2009) The repeatability of behaviour: a meta-analysis. Anim Behav 77:771-783

Budaev SV (2010) Using principal components and factor analysis in animal behaviour research: caveats and guidelines. Ethology 116:472-480

Cote J, Dreiss A, Clobert J (2008) Social personality trait and fitness. Proc R Soc Lond B 275:2851-2858

Croft DP, James R, Krause J (2008) Exploring animal social networks. Princeton University Press, Princeton

Croft DP, Krause J, Darden SK, Ramnarine IW, Faria JJ, James R (2009) Behavioural trait assortment in a social network: patterns and implications. Behav Ecol Sociobiol 63:1495-1503

Croft DP, Madden JR, Franks DW, James R (2011) Hypothesis testing in animal social networks. Trends Ecol Evol 26:502-507

Darden SK, James R, Ramnarine IW, Croft DP (2009) Social implications of the battle of the sexes: sexual harassment disrupts female sociality and social recognition. Proc R Soc Lond B 276:26512656

Dingemanse NJ, Wolf M (2010) Recent models for adaptive personality differences: a review. Philos T R Soc B 365:3947-3958

Dingemanse NJ, Dochtermann N, Wright J (2010a) A method for exploring the structure of behavioural syndromes to allow formal comparison within and between data sets. Anim Behav 79:439450

Dingemanse NJ, Kazem AJN, Réale D, Wright J (2010b) Behavioural reaction norms: animal personality meets individual plasticity. Trends Ecol Evol 25:81-89

Dingemanse NJ, Dochtermann N, Nakagawa S (2012) Defining behavioural syndromes and the role of "syndrome deviation" in understanding. Behav Ecol Sociobiol. doi:10.1007/s00265-012-1416-2

Dingemanse NJ, Dochtermann NA (2012) Quantifying individual variation in behaviour: mixed-effect modelling approaches. J Anim Ecol. doi:10.1111/1365-2656.12013

Dochtermann NA, Jenkins SH (2007) Behavioural syndromes in Merriam's kangaroo rats (Dipodomys merriami): a test of competing hypotheses. Proc R Soc Lond B 274:2343-2349

Dochtermann NA, Jenkins SH (2011) Multivariate methods and small sample sizes. Ethology 117:95-101

Drewe JA (2010) Who infects whom? Social networks and tuberculosis transmission in wild meerkats. Proc R Soc Lond B 277:633642

Ellison NB, Steinfield C, Lampe C (2007) The benefits of Facebook "friends:" social capital and college students' use of online social network sites. J Comput-Mediat Comm 12:1143-1168

Flack JC, Girvan M, de Waal FBM, Krakauer DC (2006) Policing stabilizes construction of social niches in primates. Nature 439:426-429

Fowler JH, Dawes CT, Christakis NA (2009) Model of genetic variation in human social networks. P Natl Acad Sci USA 106:17201724

Franz M, Nunn CL (2009) Network-based diffusion analysis: a new method for detecting social learning. Proc R Soc Lond B 276:1829-1836

Frere CH, Kruetzen M, Mann J, Connor RC, Bejder L, Sherwin WB (2010) Social and genetic interactions drive fitness variation in a free-living dolphin population. P Natl Acad Sci USA 107:1994919954

Hadfield JD, Wilson AJ, Garant D, Sheldon BC, Kruuk LEB (2010) The misuse of BLUP in ecology and evolution. Am Nat 175:116125

Hamede RK, Bashford J, McCallum H, Jones M (2009) Contact networks in a wild Tasmanian devil (Sarcophilus harrisii) population: using social network analysis to reveal seasonal variability in social behaviour and its implications for transmission of devil facial tumour disease. Ecol Lett 12:1147-1157

Horne TJ, Ylonen H (1998) Heritabilities of dominance-related traits in male bank voles (Clethrionomys glareolus). Evolution 52:894-899

Jacoby DMP, Busawon DS, Sims DW (2010) Sex and social networking: the influence of male presence on social structure of female shark groups. Behav Ecol 21:808-818

Krause J, Ruxton GD (2002) Living in groups. Oxford University Press, Oxford

Krause J, Croft DP, James R (2007) Social network theory in the behavioural sciences: potential applications. Behav Ecol Sociobiol 62:15-27

Krause J, James R, Croft DP (2010) Personality in the context of social networks. Philos T R Soc B 365:4099-4106

Krause J, Wilson ADM, Croft DP (2011) New technology facilitates the study of social networks. Trends Ecol Evol 26:5-6

Lea AJ, Blumstein DT, Wey TW, Martin JGA (2010) Heritable victimization and the benefits of agonistic relationships. P Natl Acad Sci USA 107:21587-21592

Lusseau D, Newman MEJ (2004) Identifying the role that animals play in their social networks. Proc R Soc Lond B 271:S477-S481

Madadhain J, Fisher D, Smyth P, White S, Boey YB (2005) Analysis and visualization of network data using JUNG. J Stat Softw 10:1-35

Manly BFJ (2007) Randomization, bootstrap, and Monte Carlo methods in biology, 3rd edn. Chapman and Hall, Boca Raton

McDonald DB (2007) Predicting fate from early connectivity in a social network. P Natl Acad Sci USA 104:10910-10914

McGhee KE, Travis J (2010) Repeatable behavioural type and stable dominance rank in the bluefin killifish. Anim Behav 79:497-507

Moore AJ (1990) The inheritance of social dominance, mating behaviour and attractiveness to mates in male Nauphoeta cinerea. Anim Behav 39:388-397

Newman MEJ (2003) The structure and function of complex networks. Siam Rev 45:167-256

Oh KP, Badyaev AV (2010) Structure of social networks in a passerine bird: consequences for sexual selection and the evolution of mating strategies. Am Nat 176:E80-E89

Otterstatter MC, Thomson JD (2007) Contact networks and transmission of an intestinal pathogen in bumble bee (Bombus impatiens) colonies. Oecologia 154:411-421

Pike TW, Samanta M, Lindstrom J, Royle NJ (2008) Behavioural phenotype affects social interactions in an animal network. Proc R Soc Lond B 275:2515-2520

Réale D, Reader SM, Sol D, McDougall PT, Dingemanse NJ (2007) Integrating animal temperament within ecology and evolution. Biol Rev 82:291-318

Ryder TB, McDonald DB, Blake JG, Parker PG, Loiselle BA (2008) Social networks in the lek-mating wire-tailed manakin (Pipra filicauda). Proc R Soc Lond B 275:1367-1374

Shizuka D, McDonald DB (2012) A social network perspective on measurements of dominance hierarchies. Anim Behav 83:925-934

Sih A, Watters JV (2005) The mix matters: behavioural types and group dynamics in water striders. Behaviour 142:1417-1431

Sih A, Bell A, Johnson JC (2004) Behavioral syndromes: an ecological and evolutionary overview. Trends Ecol Evol 19:372-378

Sih A, Hanser SF, McHugh KA (2009) Social network theory: new insights and issues for behavioral ecologists. Behav Ecol Sociobiol 63:975-988

Sih A, Cote J, Evans M, Fogarty S, Pruitt J (2012) Ecological implications of behavioural syndromes. Ecol Lett 15:278-289

Webster MM, Ward AJW (2011) Personality and social context. Biol Rev 86:759-773

Wey T, Blumstein DT, Shen W, Jordan F (2008) Social network analysis of animal behaviour: a promising tool for the study of sociality. Anim Behav 75:333-344 
Wilson ADM, Krause J (2012a) Metamorphosis and animal personality: a neglected opportunity. Trends Ecol Evol 27:529-531

Wilson ADM, Krause J (2012b) Personality and metamorphosis: is behavioral variation consistent across ontogenetic niche shifts? Behav Ecol. doi:10.1093/beheco/ars123

Wilson ADM, Godin J-GJ, Ward AJW (2010a) Boldness and reproductive fitness correlates in the eastern mosquitofish, Gambusia holbrooki. Ethology 116:96-104
Wilson AJ, Réale D, Clements MN, Morrissey MM, Postma E, Walling CA, Kruuk LEB, Nussey DH (2010b) An ecologist's guide to the animal model. J Anim Ecol 79:13-26

Wolf M, Weissing FJ (2010) An explanatory framework for adaptive personality differences. Philos T R Soc B 365:39593968

Wolf M, Weissing FJ (2012) Animal personalities: consequences for ecology and evolution. Trends Ecol Evol 27:452-461 